\title{
A study of cerium extraction by TBP and TODGA using a rotating diffusion cell
}

Michael A. Bromley, Colin Boxall

Abstract. For the study of the coupled interfacial-mass transfer kinetics of, inter alia, TBP, TODGA, CyMe ${ }_{4}-\mathrm{BTBP}$ and $\mathrm{CyMe}_{4}$-BTPhen based solvent extraction processes, a new rotating diffusion cell (RDC) apparatus has been established at Lancaster University. RDC studies of Ce(IV)/TBP and Ce(III)/TODGA extraction systems have been undertaken in order to improve the understanding of the chemical and kinetic processes involved. In each case, an interesting dependency on local hydrodynamics at the solution phase boundary with results suggesting that the organic extractant molecules migrate into the aqueous phase in order to capture Ce.

Key words: SACSESS • TBP • TODGA • rotating diffusion cell (RDC) • separation • extraction

M. A. Bromley, C. Boxall ${ }^{\bowtie}$

The LRF Centre for Nuclear Engineering,

Engineering Department,

Lancaster University,

Gillow Ave., Lancaster, LA1 4YW, United Kingdom, Tel.: +44(0)1524 594866, Fax: +44(0)1524 381707, E-mail: c.boxall@lancaster.ac.uk

Received: 19 June 2015

Accepted: 31 August 2015

\section{Introduction}

Nuclear power is key to the energy production strategy within the EU and requires exceptional attention to safety for the management of long-lived radioactive waste. As such, there is significant research interest in the development of waste handling strategies, including that of multidisciplinary consortia such as the European Commission funded SACSESS program (Safety of Actinide Separation Processes) [1] which comprises several European universities, nuclear research bodies and industrial stakeholders.

With a focus on partitioning and transmutation [2], SACSESS aims to improve the safety of long-lived radioactive waste management by reducing the amount, radiotoxicity and thermal power of the waste to be handled, thus aiding disposal via geological repository. Several aqueous partitioning processes have been developed to scientific feasibility in recent years, notably during the preceding European Commission funded program, ACSEPT (Actinide reCycling by SEParation and Transmutation) [3]. These processes often involve the use of new extractant or complexing organic molecules and diluents and must therefore be comprehensively studied to establish a clear understanding of the incorporated chemical process and associated safety issues before they can be implemented.

As a contribution to the SACSESS program, Lancaster University, UK has undertaken a study to improve the understanding of the chemical 
processes developed during ACSEPT. To facilitate this, a rotating diffusion cell (RDC) [4] apparatus has been established to enable the study of coupled interfacial-mass transfer kinetics of several organic extractant molecules which are to be studied including TBP, TODGA [5, 6], CyMe ${ }_{4}$-BTBP [7] and $\mathrm{CyMe}_{4}$-BTPhen [8]. The RDC is derived from the Lewis cell but whereas the Lewis design comprises two directly interfaced liquid phases within a single container and utilizes individual stirrers within each phase to generate interfacial flux, the RDC includes two separate compartments into which the two solution phases are placed. An inner rotating cylinder, containing the organic solution phase, and an outer non-rotating reaction vessel, containing the aqueous solution phase, are separated by a porous membrane filter mounted at the lower open end of the inner cylinder, Fig. 1 . The mounted nitrocellulose membrane (Millipore GSWP04700) is treated with clearing solvent (see experimental section) to selectively collapse the porosity around the membrane perimeter while preserving a central porous disc. This disc, which must be positioned within the controlled flow region at the centre of the cylinder (see below), then provides an interface of defined area between the two solution phases, across which the transport of species can be studied.

Successful operation of the RDC relies on the generation of hydrodynamic flow at each side of the interface membrane [4]; this is achieved by rotation of the inner cylinder via motor driven pulley system. At the outer membrane surface, this rotation generates desirable hydrodynamics which match those of widely studied rotating disc systems without modification, however, the solution in the inner cylinder is subjected to significant centrifugal force and a PTFE baffle must be employed to regulate the hydrodynamic flow. Consisting of a static cylinder with flow cycle-enabling slots in either side, the baffle is positioned centrally within the rotating inner cylinder with $\sim 2 \mathrm{~mm}$ clearance between the lower baffle edge and the interface membrane. The

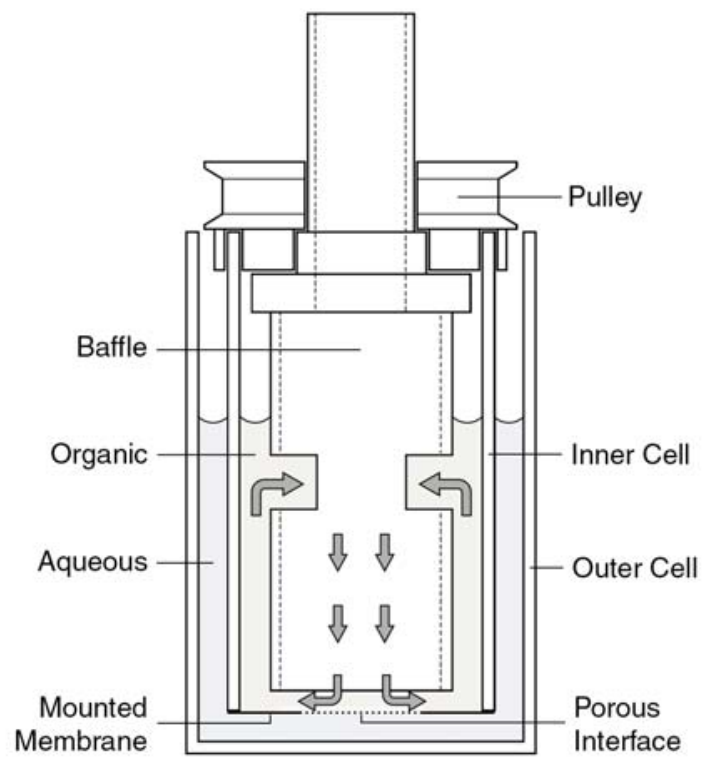

Fig. 1. Rotating diffusion cell. baffle walls isolate the centre of the rotated solution and generate a flow pattern towards and normal to the interface. Centrifugal force causes the solution to exit through the $2 \mathrm{~mm}$ gap, generating rotating disc-like hydrodynamics across the inner membrane surface. As the solution is forced up the sides of the rotating cylinder, the baffle slots allow flow back into the centre and the flow cycle is maintained.

By establishing this hydrodynamic environment at each side of the solution phase interface, the RDC enables the monitoring of both physical and chemical kinetics based on kinetic selectivity, through a high sheer, low residence environment, thermodynamic selectivity, through a low sheer, high residence environment, and the transition between the two regimes [9].

In order to gain understanding of the RDC system and to produce data on extraction systems representative of those being developed within ACSEPT and SACSESS, initial studies using the RDC have been performed on two solvent extraction systems; the well-established extraction of Ce(IV) by TBP, and the less widely studied extraction of Ce(III) by TODGA $[10,11]$. With the former being more widely understood than the latter, the TBP system will serve as a reference point for further TODGA studies. This paper will now discuss these initial results.

\section{Experimental}

All reagents used are AnalaR grade or higher, and purchased from Sigma-Aldrich (Gillingham, Dorset, UK) or Alfa Aesar (Heysham, Lancashire, UK) with the exception of TODGA (Technocomm Ltd., Wellbrae, Falkland, Scotland). All water used is ultrapure doubly de-ionized water from a Direct-Q 3 UV Millipore water purification system (Millipore, Watford, UK) to a resistivity of $18.2 \mathrm{M} \Omega \cdot \mathrm{cm}$.

Millipore GSWP04700 nitrocellulose membranes (Millipore, Watford, UK) with $0.22 \mu \mathrm{m}$ pore size and $150 \mu \mathrm{m}$ thickness are mounted on the inner Perspex RDC cylinder using methyl-methacrylate-based adhesive. The adhesive is applied to the perimeter of the membrane, encasing the membrane edge in Perspex and resulting in a highly robust and stable mounting.

Mounted interface membranes are treated with a clearing solvent (33\% $n$-hexane, $33 \% 1,2$-dichloroethane, $33 \%$ 1,4-dioxane, $1 \%$ water) in order to selectively and irreversibly collapse the porous structure of the membrane and produce a defined area porous interface, Fig. 2. Solvent is applied via micro pipette while the membrane is rotated at $\sim 20 \mathrm{~Hz}$; beginning $10 \mathrm{~mm}$ from the centre and moving radially outwards a central porous disc of $20 \mathrm{~mm}$ diameter is formed. Treated areas become transparent, completely non-porous and contract to hold the membrane under tension, ensuring stability during rotation.

Each of the aqueous solution phases of $10 \mathrm{mM}$ $\mathrm{Ce}(\mathrm{III})_{2}\left(\mathrm{SO}_{4}\right)_{3} \cdot 8 \mathrm{H}_{2} \mathrm{O}$ and $10 \mathrm{mM} \mathrm{Ce}(\mathrm{IV})\left(\mathrm{SO}_{4}\right)_{2}$ are prepared in $1 \mathrm{M}$ nitric acid while the respective organic phases consist of $0.2 \mathrm{M}$ TODGA in $5 \%$ 


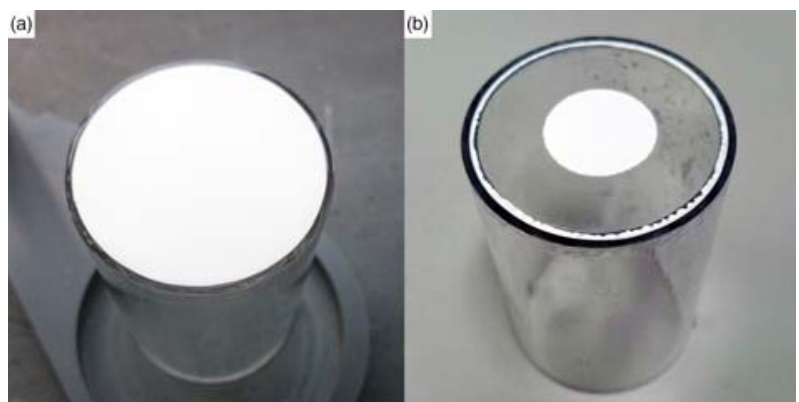

Fig. 2. Mounted nitrocellulose membrane; (a) pre-treatment and (b) post-treatment with clearing solvent.

octanol: $95 \%$ kerosene and $0.2 \mathrm{M}$ TBP in kerosene. All solutions are freshly prepared prior to each experimental run. (Future studies will be conducted using nitrate salts as opposed to sulfates in order to minimize the number of ionic species in the aqueous phase).

Once prepared, the organic solution phase is placed into the inner RDC cylinder, via a sampling port in the baffle shaft, and allowed to fully permeate the nitrocellulose membrane. The aqueous solution is placed into the outer glass vessel and raised to submerge the inner cylinder until the two solution levels are equal. This sequence ensures that the phase boundary is positioned at the outer membrane surface.

The RDC is then operated at a fixed rotation speed between $0-10 \mathrm{~Hz}$ for $120 \mathrm{~min}$ with monitoring of each solution phase performed using a Gamry Spectro-115U UV-Vis spectrometer (Gamry Instruments, Cheshire, UK). UV-Vis absorbance measurements are taken from the aqueous phase at 0 and $120 \mathrm{~min}$ and from the organic phase, via the baffle sample port, at $15 \mathrm{~min}$ intervals without disturbing the rotation.

\section{Results and discussion}

Each of the aforementioned extraction systems was studied in the rotating diffusion cell as a function of rotation speed. In the case of the Ce(IV)/TBP system, the aqueous phase comprised $10 \mathrm{mM} \mathrm{Ce}$ (IV) in $1 \mathrm{M}$ nitric acid with $0.2 \mathrm{M}$ TBP in kerosene as the organic phase. Extraction was monitored at rotation speeds of $0,1.0,2.5,5.0$ and $10 \mathrm{~Hz}$; at each speed, UV-Vis absorbance measurements of the organic phase demonstrate a peak absorbance at $335 \mathrm{~nm}$, indicating the presence of a $\mathrm{Ce}(\mathrm{IV}) / \mathrm{TBP}$ complex, which increases with time at a steady rate. Representative data recorded at a rotation speed of $5.0 \mathrm{~Hz}$ is shown in Fig. 3.

Analysis of the peak absorbance as a function of time reveals that the $\mathrm{Ce}(\mathrm{IV}) / \mathrm{TBP}$ extraction system proceeds with a linear rate and shows no evidence of saturation over the experimental period, Fig. 4. However, it can be seen that the peak absorbance, and hence the $\mathrm{Ce}(\mathrm{IV})$ concentration, decreases with increasing rotation speed. This may initially appear to be counter-intuitive as it is not unreasonable to assume that as the rotation speed of the RDC is increased, so too is the hydrodynamic flow within

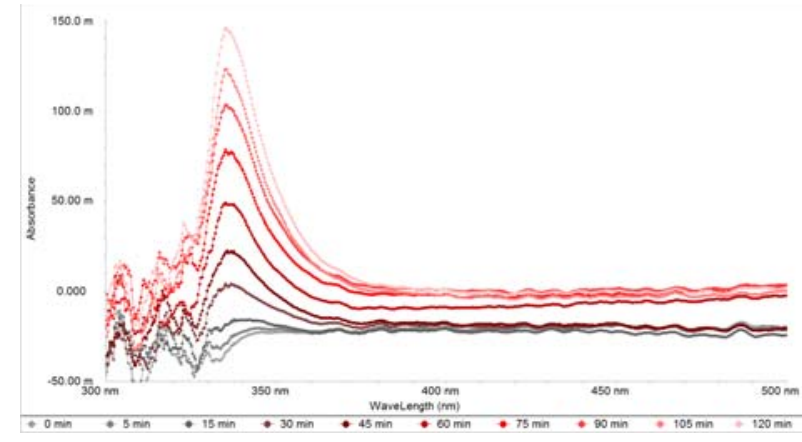

Fig. 3. UV-Vis absorbance spectra of $\mathrm{Ce}(\mathrm{IV}) / \mathrm{TBP}$ system organic phase at $5 \mathrm{~Hz}$ rotation speed.

the cell; consequently, the flux of species towards the interface would also be expected to be increased and contact between the two solution phases enhanced, leading to an increased rate of extraction. Indeed this is true for low rotation speeds when compared to the rate of extraction during no rotation. However, this effect is reversed as rotation speed is further increased.

These results suggest that the high solution flux is preventative of Ce extraction, perhaps due to a reduction in residence time, a local hydrodynamic effect at the interface, or a combination of both factors. While the former does seem to be a logical contributor, it is the possible interface hydrodynamic effects which should draw most interest. As previously discussed, the RDC membrane is under hydrodynamic conditions comparable to those at a rotating disc electrode (RDE). It is known from existing (RDE) studies that a diffusion layer exists at the surface of the rotating disc. The thickness of this layer, $X_{D}$, is given by the Levich equation [12], where $\mathrm{D}$ is the diffusion coefficient, $v$ is the kinetic viscosity and $\omega$ is rotation speed in $\mathrm{Hz}$, which shows that $\mathrm{X}_{\mathrm{D}}$ decreases with increasing rotation speed.

$$
\mathrm{X}_{\mathrm{D}}=0.643 \mathrm{D}^{1 / 3} v^{1 /} \omega^{-1 / 2}
$$

In the context of a cylindrical polar co-ordinate system with its origin at the centre of the rotating disc surface, the solution within the diffusion layer may be considered to rotate with the disc and be free from any convective mass transport either radially or in the direction normal to the rotating disc surface. A similar phenomenon will be observed at the RDC

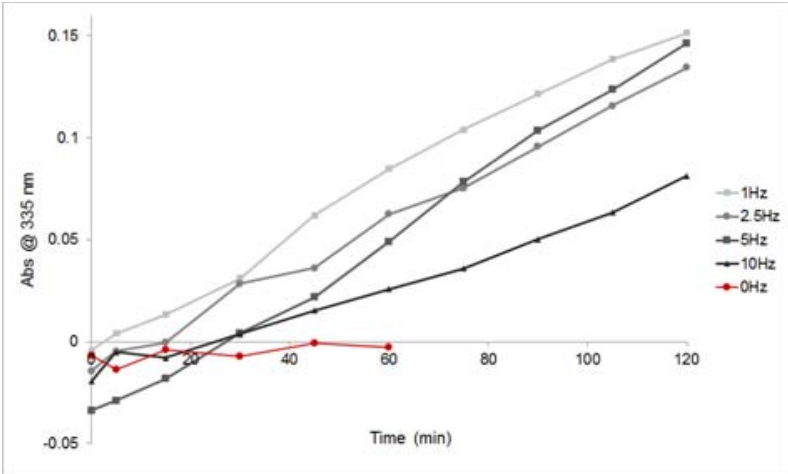

Fig. 4. Peak absorbance at $335 \mathrm{~nm}$ for $\mathrm{Ce}(\mathrm{IV}) / \mathrm{TBP}$ system organic phase vs. time; rotation speeds $0-10 \mathrm{~Hz}$. 


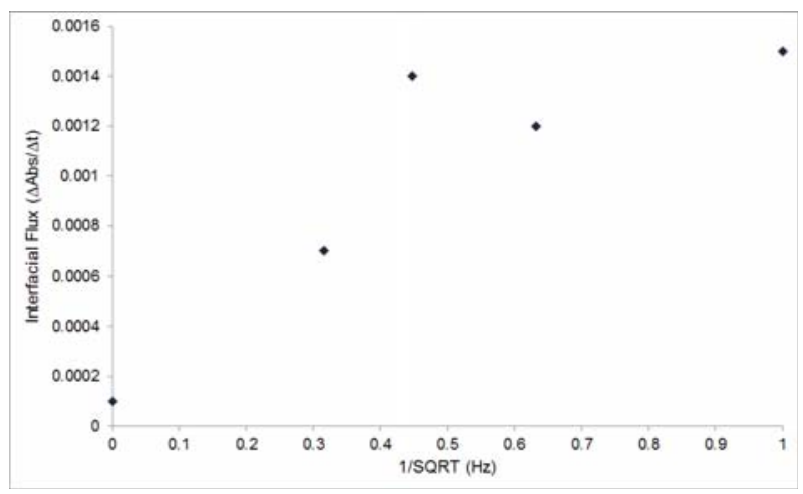

Fig. 5. Interfacial flux vs. inverse square root of rotation speed for Ce(IV)/TBP system.

interface and this may be an influencing factor on the rate of extraction.

Further analysis confirms a relationship between the extraction rate and rotation speed; plotting the interfacial flux, as given by the slope of each plot in Fig. 4, against the inverse of the square root of rotation speed, an approximately linear trend is seen, Fig. 5, particularly if the outlying $5.0 \mathrm{~Hz}$ result is disregarded.

Considering that both the diffusion layer thickness and the rate of extraction have an inverse relationship with rotation speed, it is implied that the capture of $\mathrm{Ce}$ (IV) by TBP is occurring within a diffusion layer. Furthermore, we are able to surmise in which phase this capture is occurring by considering the location of the diffusion layer at either side of the phase boundary. Given that the RDC has been assembled in such a way to ensure that the nitrocellulose interface membrane is saturated with the organic phase and that, as a result, the phase boundary is positioned at the outer membrane surface, the organic phase diffusion layer thickness, $\mathrm{X}_{\mathrm{D}}^{\mathrm{org}}$, which according to Eq. (1) will be of the order of less than $100 \mu \mathrm{m}$, will lie within the $150 \mu \mathrm{m}$ thickness of the interface membrane where it is shielded from the effects of rotation speed. Conversely, the aqueous phase diffusion layer, $\mathrm{X}_{\mathrm{D}}^{\mathrm{aq}}$, lies outside the membrane structure and hence is not protected from the effects of rotation, thus remaining susceptible to speed variation. From the dependence of Ce concentration on rotation speed, it can be concluded that Ce capture is not only occurring within a diffusion layer at the membrane surface but that it is specifically occurring within the aqueous phase diffusion layer. This implies that the extractant is migrating into the aqueous solution in order to form a complex with Ce before returning back across the interface to the organic phase.

The route by which a reduction in the diffusion layer thickness affects the extraction rate can be qualitatively considered as follows. If it is assumed that the organic extractant is indeed migrating into the aqueous solution phase, a concentration gradient is established across the aqueous diffusion layer with extractant concentration decreasing linearly with distance from the phase boundary, Fig. 6 . A greater diffusion layer thickness, $X_{D}$, results in a greater penetration depth of the extractant into the aqueous phase and hence greater accessibility

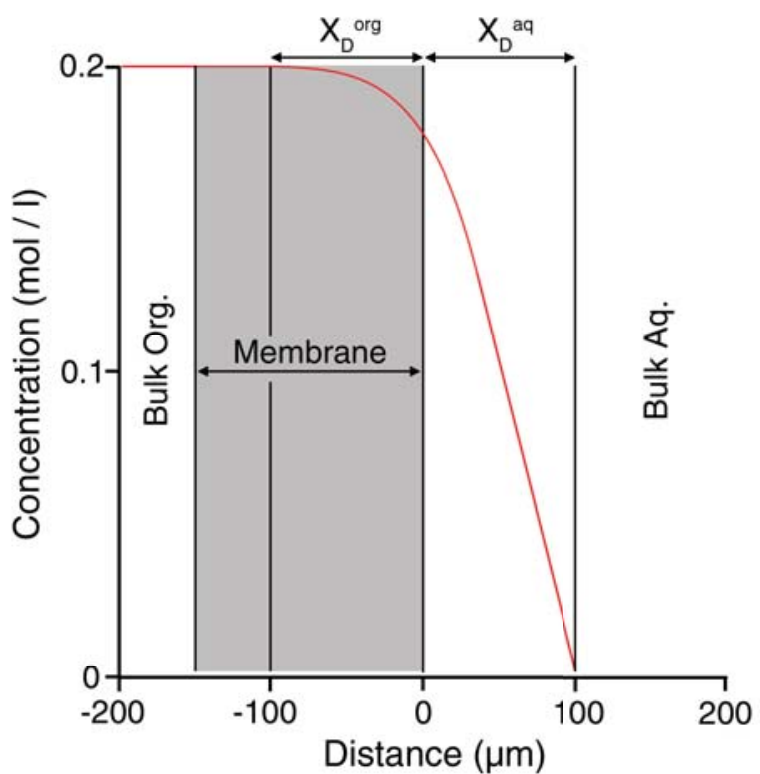

Fig. 6. Schematic of organic, $X_{D}^{\text {org }}$, and aqueous, $X_{D}^{\text {aq }}$, diffusion layer positioning and extractant concentration profile.

to free Ce for capture. As $\mathrm{X}_{\mathrm{D}}^{\mathrm{aq}}$ is reduced, so is the contact between the complexing species, and the rate of extraction drops accordingly.

In light of the above qualitative understanding, the less well-studied TODGA extractant molecule was investigated with the RDC, again as a function of rotation speed. In this case the aqueous phase comprised $10 \mathrm{mM} \mathrm{Ce}(\mathrm{III})$ in $1 \mathrm{M}$ nitric acid with $0.2 \mathrm{M}$ TODGA in 5\% octanol:95\% kerosene as the organic phase. Extraction was monitored at rotation speeds of $0,1.0,2.5,5.0,7.5$ and $10 \mathrm{~Hz}$; at each speed, UV-Vis absorbance measurements of the organic phase demonstrate a peak absorbance at $343 \mathrm{~nm}$, representative of a Ce(III)/TODGA complex, which increases with time. Representative data recorded at a rotation speed of $7.5 \mathrm{~Hz}$ is shown in Fig. 7.

Due to the greater hydrophobicity of TODGA, in comparison to TBP, it may be expected that the extractant would not migrate into the aqueous solution phase and hence not display the same dependency on rotation speed as observed in the Ce(IV)/TBP system above. However, by plotting the peak absorbance as a function of time for each rotation speed, Fig. 8, it can be seen that the rate of extraction in the Ce(III)/TODGA system also decreases with increasing rotation. Paradoxically,

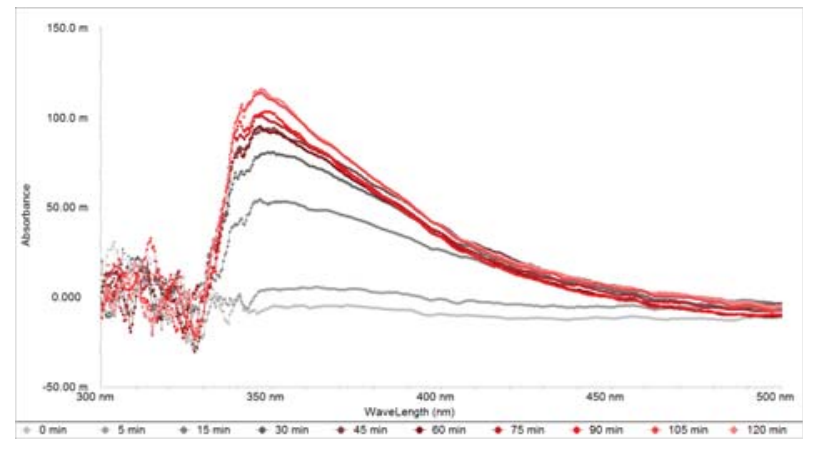

Fig. 7. UV-Vis absorbance spectra of Ce(III)/TODGA system organic phase at $5 \mathrm{~Hz}$ rotation speed. 


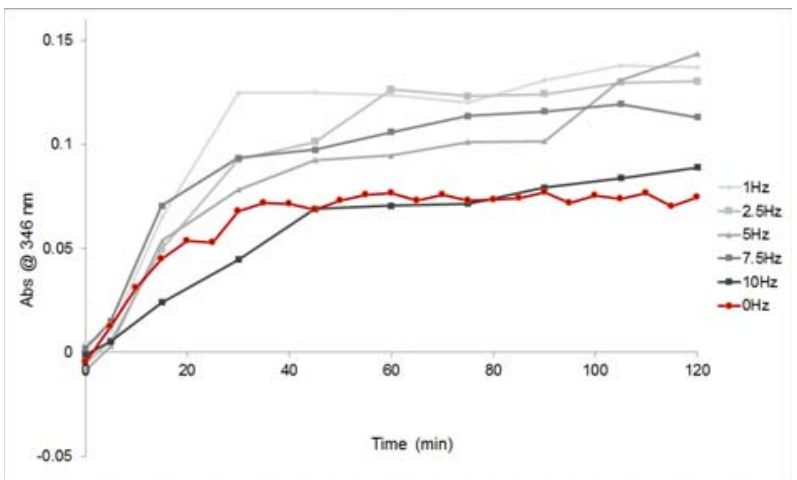

Fig. 8. Peak absorbance at $346 \mathrm{~nm}$ for Ce(III)/TODGA system organic phase vs. time; rotation speeds $0-10 \mathrm{~Hz}$.

while the solution flux generated by rotation does appear to improve extraction rates at low rotation speeds, the concentration of Ce(III) transferred at high rotation speeds is approximately equal to that observed during experiments on static membranes.

If these observations are met with the same reasoning as applied to the Ce(IV)/TBP system, it would suggest that the Ce(III)/TODGA system is also susceptible to local hydrodynamic effects at the interface and that, as with $\mathrm{Ce}$ (IV), $\mathrm{Ce}$ (III) capture is occurring within the aqueous diffusion layer. This clearly implies that TODGA is migrating across the interface and into the aqueous solution phase and represents an unexpected finding.

Further interest is seen in the fact that, unlike the Ce(IV)/TBP system which progresses linearly with no evidence of saturation, the Ce(III)/TODGA system appears to plateau at times greater than $40 \mathrm{~min}$. While this could initially be interpreted to indicate that total Ce extraction is being achieved, this is known to be untrue with UV-Vis measurements of the aqueous phase (not shown) suggesting that $\mathrm{Ce}$ (III) concentration changes are proportionally negligible at $t=120 \mathrm{~min}$, hence an excess of $\mathrm{Ce}$ (III) remains available for extraction. It may be hypothesized then that the plateau effect is indicative of a lower D value for the Ce(III)/TODGA system than for the $\mathrm{Ce}(\mathrm{IV}) / \mathrm{TBP}$ system or perhaps that a saturation point wherein a limit to the amount of TODGA entering the aqueous diffusion layer is being reached. The latter theory is compatible with the high hydrophobicity of the molecule although the system requires further investigation and understanding before a definitive conclusion can be reached.

\section{Conclusion}

A rotating diffusion cell system has been established at Lancaster University and commissioned for the study of the coupled interfacial - mass transfer kinetics of several organic extractant molecules relevant to the SANEX and PUREX processes. Using the RDC, the extraction of $\mathrm{Ce}(\mathrm{IV})$ and $\mathrm{Ce}(\mathrm{III})$ from nitric acid by TBP and TODGA, respectively have been investigated at a range of rotation speeds.

Despite expectations that an increase in RDC rotation speed, and the associated solution flux to- wards the interface, would result in an increased rate of extraction through improved solution contact, the opposite has been observed. In both the Ce(IV)/ TBP and Ce(III)/TODGA systems the extraction rate displays an inverse relationship with rotation speed, indicating that each process is affected by local hydrodynamics. It is known that the RDC operates with the same hydrodynamic principals as a rotating disc electrode and hence that a diffusion layer is generated at the rotating surface. The thickness of this layer shares the same inverse relationship to rotation speed so it is implied that complexation of Ce by the extractant molecules occurs within a hydrodynamically generated diffusion layer. The assembly of the RDC is such that the solution phase boundary is positioned at the outer membrane surface and hence any diffusion layer generated in the organic phase is placed within the interface membrane, and hence is shielded from the effects of rotation speed increases, while a diffusion layer in the aqueous phase is not. Given that the rate of extraction is susceptible to rotation speed increase and the consequent reduction in diffusion layer thickness, it is determined that the key interaction between Ce and extractant molecules occurs on the aqueous side of the phase boundary.

The findings clearly imply that each of the studied extractant molecules must migrate across the boundary and into the aqueous solution phase. While this may be anticipated for TBP which is known to enter the aqueous phase, it certainly represents and unforeseen outcome which changes the understanding of the behavior of TODGA in a solvent extraction system.

Ongoing studies are aiming to interrogate the influence of variables such as aqueous Ce concentration and acidity as well as to quantitatively determine the amount of $\mathrm{Ce}$ which is extracted, thus enabling the calculation of the distribution-coefficient.

Acknowledgments. SACSESS is supported by the European Commission under the Euratom Nuclear Fission and Radiation Protection Theme of the 7th Framework Programme for Research \& Technological Development (FP7 EURATOM Fission-2012-2.3.1 Project Reference no. 323282). We would also like to thank The Lloyd's Register Foundation (LRF) for supporting research facilities at the Lancaster University. The Lloyd's Register Foundation helps to protect life and property by supporting engineering-related education, public engagement and the application of research.

\section{References}

1. Bourg, S. (2015). SACSESS - Safety of actinide separation processes. Available from http://www.sacsess.eu/

2. Basak, U., \& Dyck, G. R. (2010). IAEA activities on assessment of partitioning processes for transmutation of actinides. In Proceedings of the First ACSEPT International Workshop, 31 March - 2 April 2010. Lisbon, Portugal.

3. Bourg, S., Touron, E., Caravaca, C., Ekberg, C., Gaubert, E., \& Hill, C. (2008). ACSEPT: a new FP7- 
Euratom Collaborative Project in the field of partitioning processes for advanced fuel cycles. In ATALANTE 2008, May 19-22. Montpellier.

4. Albery, W. J., Burke, J. F., Leffler, E. B., \& Hadgraft, J. (1976). Interfacial transfer studied with a rotating diffusion cell. J. Chem. Soc. Faraday Trans. 1, 72, 1618-1626. DOI: 10.1039/f19767201618.

5. Modolo, G., Asp, H., Schreinemachers, C., \& Vijgen, H. (2007). Development of a TODGA based process for partitioning of actinides from a PUREX raffinate Part I: Batch extraction optimization studies and stability tests. Solvent Extr. Ion Exch., 25, 703-721. DOI: $10.1080 / 07366290701634578$

6. Sypula, M., Wilden, W., Schreinemachers, C., \& Modolo, G. (2010). Separation of An(III) from PUREX raffinate as an innovative SANEX process based on a mixture of TODGA/TBP. In Proceedings of the First ACSEPT International Workshop, 31 March - 2 April 2010. Lisbon, Portugal.

7. Löfström-Engdahl, E., Aneheim, E., Ekberg, C., Foreman, M., Halleröd, J., \& Skarnemark, G. (2014). Extraction thermodynamics of $\mathrm{Am}$ (III) and $\mathrm{Eu}$ (III) using $\mathrm{CyMe}_{4}$-BTBP in various organic diluents. J. Chem. Therm., 76, 64-69. DOI: 10.1016/j.jct.2014.03.004.
8. Afsar, A., Harwood, L. M., \& Hudson, M. J. (2015). The development of molecules for the highly efficient separation of americium(III) from lanthanides(III). In Reference module in chemistry, molecular sciences and chemical engineering. Elsevier. Retrieved from http://www.sciencedirect.com/science/article/pii/ B9780124095472113988.

9. Albery, W. J., \& Choudhery, R. A. (1988). Transport and kinetics in reactions involving two liquid phases. J. Phys. Chem., 92, 1151-1155. DOI: 10.1021/ j100316a030.

10. Wilden, A., Modolo, G., Sypula, M., Geist, A., \& Magnusson, D. (2012). The recovery of An(III) in an innovative-SANEX process using a TODGA-based solvent and selective stripping with a hydrophilic BTP. Procedia Chem., 7, 418-424. DOI: 10.1016/j. proche.2012.10.065.

11. Sharrad, C. A., \& Whittaker, D. M. (2015). The use of organic extractants in solvent extraction processes in the partitioning of spent nuclear fuels. In R. Taylor (Ed.), Reprocessing and recycling of spent nuclear fuel (pp. 153-189). Oxford: Woodhead Publishing.

12. Levich, V. G. (1962). Physicochemical hydrodynamics. Englewood Cliffs, N.J.: Prentice-Hall. 